\title{
POLYTOPE VOLUME COMPUTATION
}

\author{
JIM LAWRENCE
}

\begin{abstract}
A combinatorial form of Gram's relation for convex polytopes can be adapted for use in computing polytope volume. We present an algorithm for volume computation based on this observation. This algorithm is useful in finding the volume of a polytope given as the solution set of a system of linear inequalities, $P=\left\{x \in \mathbb{R}^{n}: A x \leq b\right\}$.

As an illustration we compute a formula for the volume of a projective image of the $n$-cube. From this formula we deduce that, when $A$ and $b$ have rational entries (so that the volume of $P$ is also a rational number), the number of binary digits in the denominator of the volume cannot be bounded by a polynomial in the total number of digits in the numerators and denominators of entries of $A$ and $b$. This settles a question posed by Dyer and Frieze.
\end{abstract}

\section{INTRODUCTION}

We present a method for computing exactly the volume of a convex polytope given as the set of solutions of a finite system of linear inequalities.

Some methods for exact computation of the volume of a convex polytope $P$ in $\mathbb{R}^{n}$ are given in [1, 5, 13,30]. In Cohen and Hickey [5] and Von Hohenbalken [30], the volume is obtained by triangulating the polytope and summing the volumes of the simplexes of the triangulation. (Cohen and Hickey [5] compare this method with an approximate method.) In Allgower and Schmidt [1], the volume is computed from a triangulation of the boundary of $P$. Lasserre [13] presents a method based on the recursive use of a well-known formula for the volume (Theorem 37 of [8]); in many cases this approach also amounts to summing the volumes of the simplexes in a certain triangulation of the polytope. The method in the present paper avoids triangulation of $P$ or of its boundary.

Several papers concern computing the volume of certain sets in $\mathbb{R}^{3}$, e.g., Lee and Requicha [15, 16], where more general three-dimensional sets are considered, and Shoemaker and Huang [26]. In Speevak [27], a novel method for computing volumes of certain pyramids in $\mathbb{R}^{n}$ is given.

The method presented in this paper is based essentially on Gram's relation (see Shephard [25]). If the polytope $P$ is simple, then Gram's relation provides a method by which one can write the volume of $P$ as a sum of numbers $N_{v}$, one for each vertex $v$ of $P$. These numbers are easy to compute, so the difficulty of the procedure is mainly that of enumerating the vertices of $P$.

Received January 15, 1990.

1980 Mathematics Subject Classification (1985 Revision). Primary 52A25. 
Recent results on the complexity of volume estimation appear in Bárány and Füredi [3], Elekes [9], and Lovász [17]. These results pertain to convex sets $C$ not necessarily given as an intersection of halfspaces, but rather determined by certain oracles, e.g., by an oracle that, when given $x \in \mathbb{R}^{n}$, either assures us that $x \in C$, or gives us a halfspace $H$ containing $C$ but not $x$.

The problem considered in this paper has been shown to be $\# P$-hard (Dyer and Frieze [7]), even when restricted to polytopes for which the coefficient matrix of the defining system of inequalities is totally unimodular. (For a treatment of "\#P-hardness" see Valiant [29].)

Also, in [7], Dyer and Frieze pose the following problem. Let $A$ be an $m \times n$ matrix of rational numbers, and let $b$ be a column vector of $m$ rational numbers. Let $P=\left\{x \in \mathbb{R}^{n}: A x \leq b\right\}$ be a bounded polytope so that the volume of $P$ will necessarily be a rational number. Define the size (as in [24]) of the rational number $r=a / b$ (reduced) to be one more than the total number of digits in the binary representations of the integers $a$ and $b$, and the size of the pair $(A, b)$ to be $m(n+1)$ more than the sum of the sizes of the entries of $A$ and $b$. Is the size of the volume of $P$ polynomially bounded in the size of $(A, b)$ ? We shall see that the answer to this question is "no."

For background material concerning convex polytopes, systems of linear inequalities, linear programming, and valuations on convex polytopes, see [11, $28,10,23]$.

\section{STATEMENT OF THE MAin Result,}

\section{AND A RESULT FROM COMBINATORIAL INTEGRAL GEOMETRY}

We identify $\mathbb{R}^{n}$ with the vector space of real column vectors of length $n$. Let $P \subseteq \mathbb{R}^{n}$ be an $n$-dimensional polyhedron. Then $P$ is the set of solutions to a finite system of linear inequalities, say, $P=\left\{x \in \mathbb{R}^{n}: a_{i}^{t} x \leq b_{i}\right.$ for $\left.1 \leq i \leq m\right\}$, where the $a_{i}$ 's are in $\mathbb{R}^{n}$ and the $b_{i}$ 's are in $\mathbb{R}$. Given such a representation, the function $r_{i}(x)=b_{i}-a_{i}^{t} x$ is called the ith residual. The polyhedron $P$ is the set on which all the residuals are nonnegative. The $i$ th inequality constraint is said to be binding at $x$ if $r_{i}(x)=0$. The result upon which our algorithm for volume computation rests is as follows:

Theorem. Suppose $P=\left\{x \in \mathbb{R}^{n}: r_{i}(x)=b_{i}-a_{i}^{t} x \geq 0\right.$ for $\left.i=1, \ldots, m\right\}$. Suppose further that $P$ is bounded and that for each vertex $v$ of $P$ the number of indices $i$ such that $r_{i}(v)=0$ is $n$. In particular, $P$ is a simple polytope. Suppose $c \in \mathbb{R}^{n}$ and $d \in \mathbb{R}$ are such that the function $f(x)=c^{t} x+d$ is nonconstant on each edge of $P$. Given a vertex $v$ of $P$, let

$$
N_{v}=\frac{f(v)^{n}}{n ! \delta_{v} \gamma_{1} \cdots \gamma_{n}}
$$

where, if the indices of the constraints which are binding at $v$ are $i_{1}, \ldots, i_{n}$, then $\gamma_{1}, \ldots, \gamma_{n}$ are such that

$$
c=\gamma_{1} a_{i_{1}}+\cdots+\gamma_{n} a_{i_{n}}
$$


and $\delta_{v}$ is the absolute value of the determinant of the $n \times n$ matrix whose columns are $a_{i_{1}}, \ldots, a_{i_{n}}$. Then the volume of $P$ is

$$
\operatorname{vol}(P)=\sum_{\substack{v, \begin{array}{c}
\text { a vertex } \\
\text { of } P
\end{array} \\
N}} N_{v}
$$

This theorem follows modulo the computation of the numbers $N_{v}$ from the corollary at the end of this section. The numbers $N_{v}$ are computed (under the unnecessary but convenient restriction that $P$ be contained in the nonnegative orthant in $\mathbb{R}^{n}$ and have the origin as a vertex) in $\S 3$.

We next describe a combinatorial form of Gram's relation.

If $v$ is a vertex of $P$, we wish to describe the "forward cone" of $P$ at $v$ (with respect to $f$ ). Let $i_{1}, \ldots, i_{n}$ be the indices of the $n$ constraints which are binding at $v$. Then $v$ is the unique solution to the system of equations

$$
a_{i_{j}}^{t} x=b_{i_{j}} \quad(j=1, \ldots, n) .
$$

It follows that $\left\{a_{i_{1}}, \ldots, a_{i_{n}}\right\}$ forms a basis for $\mathbb{R}^{n}$, and there is a unique representation $c=\sum_{j=1}^{n} \gamma_{j} a_{i_{j}}$ of $c$ in terms of the basis. From this we have $f(x)=f(v)-\sum_{j=1}^{n} \gamma_{j} r_{i_{j}}(x)$. Omitting any one of the constraints in (1) leads to a system whose solution set is a line through $v$. Each edge of $P$ containing $v$ spans such a line. Since $f$ is assumed to be nonconstant on each of the edges, it follows that $\gamma_{j} \neq 0$ for $j=1, \ldots, n$. We denote by $e(v)$ the number of indices $j$ such that $\gamma_{j}>0$. This is also the number of edges of $P$ containing $v$ on which $f$ decreases in the direction leaving $v$. The forward cone at $v$ is the set $F(v)$ of solutions $x$ to the following system of inequalities:

$$
\begin{array}{ll}
r_{i_{j}}(x)<0 & \text { if } \gamma_{j}>0, \\
r_{i_{j}}(x) \geq 0 & \text { if } \gamma_{j}<0 .
\end{array}
$$

The closure of this set is a simplicial cone with apex $v$, and on this cone $f$ achieves its minimum value at $v$.

For a set $K \subseteq \mathbb{R}^{n}, C(K)$ denotes the characteristic function of $K$, so that for $x \in \mathbb{R}^{n}$

$$
C(K)(x)= \begin{cases}1 & \text { if } x \in K, \\ 0 & \text { if } x \notin K .\end{cases}
$$

If $G$ is a face of the convex polyhedron $P$, we denote by $\gamma(G, P)$ the cone generated by $P$ at $G: \gamma(G, P)=\{g+\alpha(y-x): x, g \in G, y \in P$, and $\alpha \geq 0\}$.

Lemma. For $P \subseteq \mathbb{R}^{n}$ a simple, $n$-dimensional polyhedron, $v$ a vertex of $P$, and $f(x)=c^{t} x+d$ a function which is nonconstant on each edge of $P$, as above, we have

$$
(-1)^{e(v)} C(F(v))=\sum_{G}(-1)^{\operatorname{dim}(G)} C(\gamma(G, P)),
$$


where the summation extends over all faces $G$ of $P$ such that $f$ attains its maximum value on $G$ at $v$.

Proof. Let $P$ be given (as above) as the set of solutions to the inequalities $r_{i}(x) \geq 0 \quad(i=1, \ldots, m)$, and suppose that $i_{1}, \ldots, i_{n}$ are the indices of the constraints which are binding at $v$. The $2^{n}$ subsets of $[n]=\{1,2, \ldots, n\}$ are in bijective correspondence with the faces of $P$ containing $v$ by the rule $S \rightarrow G(S)=P \cap\left\{x \in \mathbb{R}^{n}: r_{i_{j}}(x)=0\right.$ for $\left.j \in S\right\}$, for $S \subseteq[n]$. The function $G$ is order-reversing: If $S \subseteq T$, then $G(T) \subseteq G(S)$. We have $\operatorname{dim}(G(S))=n-|S|$. Also, for $S \subseteq[n], \gamma(G(S), P)=\left\{x \in \mathbb{R}^{n}: r_{i_{j}}(x) \geq 0\right.$ for $\left.j \in S\right\}$, so that $x \in \gamma(G(S), P)$ if and only if $S \subseteq T_{x}$, where $T_{x}=\left\{j \in[n]: r_{i_{j}}(x) \geq 0\right\}$.

Suppose we have, as above, $f(x)=f(v)-\sum_{j=1}^{n} \gamma_{j} r_{i_{j}}(x)$. Let $W=\{j \in$ $\left.[n]: \gamma_{j}<0\right\}$. Then $f$ assumes its maximum value on $G(W)$ at $v$, and $W$ is the (unique) smallest such set. For $S \subseteq[n], f$ assumes its maximum value on $G(S)$ at $v$ if and only if $S \supseteq W$.

For $x \in \mathbb{R}^{n}$, the value of the right-hand side of the equation in the lemma is

$$
\begin{aligned}
& \sum_{\substack{S \subseteq[n] \\
S \supseteq \underline{W}}}(-1)^{\operatorname{dim}(G(S))} C(\gamma(G(S), P))(x) \\
& \quad=\sum_{W \subseteq S \subseteq T_{x}}(-1)^{n-|S|}= \begin{cases}(-1)^{n-|W|} & \text { if } T_{x}=W, \\
0 & \text { otherwise. }\end{cases}
\end{aligned}
$$

Clearly, this is $(-1)^{e(v)} C(F(v))(x)$.

In the proof of the theorem below we use a version of Gram's relation. (See Shephard [25]. The following is a strengthened version which can be proven using methods of [25]. Gram's relation is also known as the Brianchon-Gram Theorem. See McMullen [18].)

Gram's relation. Let $P$ be a convex polyhedron having at least one vertex. Then

$$
\sum_{\substack{G, \text { abounded } \\ \text { face of } P}}(-1)^{\operatorname{dim}(G)} C(\gamma(G, P))=C(P) .
$$

Theorem. Suppose $P$ and $f$ are as in the statement of the lemma. Additionally, assume that $f$ attains its minimum value on $P$. Then

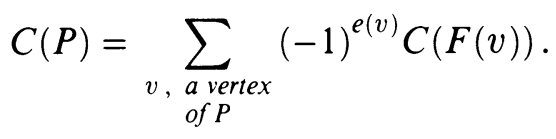

Proof. We have

$$
\begin{aligned}
\sum_{\substack{v, \text { a vertex } \\
\text { of } P}}(-1)^{e(v)} C(F(v)) & =\sum_{\substack{v \text { a vertex } \\
\text { of } P}} \sum_{\begin{array}{c}
G, \text { a face of } P \\
\text { on which } f \text { attains } \\
\text { its maximum value at } v
\end{array}}(-1)^{\operatorname{dim}(G)} C(\gamma(G, P)) \\
& =\sum_{\begin{array}{c}
G, \text { a bounded } \\
\text { face of } P
\end{array}}(-1)^{\operatorname{dim}(G)} C(\gamma(G, P))=C(P) .
\end{aligned}
$$


The first of these equalities follows from the lemma; the second from the fact that a face $G$ on which $f$ is bounded above and below must be bounded, since $f$ is not constant on any edge of $G$; and the third from the above version of Gram's relation.

This theorem is useful, as we shall see, not only in volume computation but also in the computation of any valuation which can easily be evaluated on simplexes. We recall some fundamental facts concerning valuations, beginning with the definition (see also [14]).

Let $\mathscr{F}$ be a family of sets in $\mathbb{R}^{n}$ which is closed under finite intersections and unions, and suppose $\phi \in \mathscr{F}$. A valuation on $\mathscr{F}$ is a function $V: \mathscr{F} \rightarrow \mathbb{R}$ such that (i) $V(\phi)=0$ and (ii) for each pair of sets $A, B \in \mathscr{F}$, the identity $V(A)+V(B)=V(A \cap B)+V(A \cup B)$ holds.

Any valuation $V$ on $\mathscr{F}$ induces a homomorphism $\tilde{V}: \mathscr{S}(\mathscr{F}) \rightarrow \mathbb{R}$, where $\mathscr{S}(\mathscr{F})$ is the additive group generated by the characteristic functions $C(F)$ of elements $F$ of $\mathscr{F}$, satisfying $V(F)=\widetilde{V}(C(F))$ for each $F \in \mathscr{F}$.

Here we are interested in examples in which $\mathscr{F}$ is a collection of sets which are finite unions of polyhedra. For such a collection, given a function $k$ which is integrable on each element of $\mathscr{F}$, we can define a valuation by integration: $V(F)=\int_{F} k d \mu$. (In this case, the induced homomorphism $\widetilde{V}: \mathscr{S}(\mathscr{F}) \rightarrow \mathbb{R}$ is given by $\widetilde{V}(g)=\int_{\mathbb{R}^{n}} g k d \mu$.) For $\mathscr{F}$ the collection of finite unions of convex polytopes, taking $k \equiv 1$, we get $V(F)=\operatorname{vol}(F)$, the ordinary volume of $F$.

We can now state the following corollary to the theorem.

Corollary. If $V$ is any valuation defined on a family $\mathscr{F}$ which includes the polyhedron $P$ of the theorem and all of the forward cones $F(v)$ for vertices $v$ of $P$, then

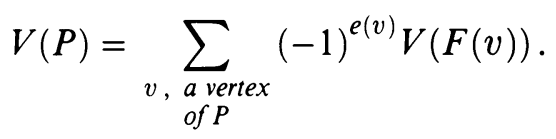

Proof. If $\tilde{V}: \mathscr{S}(\mathscr{F}) \rightarrow \mathbb{R}$ is the induced homomorphism, then we have

$$
\begin{aligned}
V(P) & =\widetilde{V}(C(P))=\widetilde{V}\left(\sum_{v, \begin{array}{c}
\text { a vertex } \\
\text { of } P
\end{array}}(-1)^{e(v)} C(F(v))\right) \\
& =\sum_{\substack{v, \text { a vertex } \\
\text { of } P}}(-1)^{e(v)} \tilde{V}(C(F(v)))=\sum_{\substack{v, \text { a vertex } \\
\text { of } P}}(-1)^{e(v)} V(F(v)) .
\end{aligned}
$$

Of course, the volume function fails to satisfy the hypothesis of this corollary because it is not defined on the (unbounded) forward cones. We may still use the corollary to evaluate $\operatorname{vol}(P)$, if $P$ is a polytope, as follows. Let $t$ be a real number large enough so that the halfspace $H_{t}=\left\{x \in \mathbb{R}^{n}: f(x) \leq t\right\}$ contains $P$. Let the valuation $V$ be defined by $V(F)=\operatorname{vol}\left(F \cap H_{t}\right)$ for any set $F$ which is the finite union of convex polyhedra whose intersections with $H_{t}$ are bounded. Now the corollary applies. The left-hand side of the equation is the 
volume of $P$. On the right-hand side is a sum involving volumes of sets of the form $F(v) \cap H_{t}$, which are simplexes.

As an example, consider the case in which $P$ is the unit $n$-cube,

$$
P=C^{n}=\left\{\left[x_{1}, \ldots, x_{n}\right]^{t} \in \mathbb{R}^{n}: 0 \leq x_{i} \leq 1 \text { for } 1 \leq i \leq n\right\} .
$$

Let $f(x)=\gamma_{1} x_{1}+\cdots+\gamma_{n} x_{n}$, where the $\gamma_{i}$ 's are positive. Let $v=\left[\varepsilon_{1} \cdots \varepsilon_{n}\right]^{t}$, where $\varepsilon_{i}=0$ or 1 for each $i$, so that $v$ is one of the $2^{n}$ vertices of $C^{n}$. The forward cone $F(v)$ is the solution set of the system

$$
\begin{array}{ll}
x_{i} \geq 0 & \text { if } \varepsilon_{i}=0, \\
x_{i}>1 & \text { if } \varepsilon_{i}=1,
\end{array}
$$

and $F(v) \cap H_{t}$ is the set which also satisfies the additional inequality $\sum_{i=1}^{n} \gamma_{i} x_{i} \leq$ $t$. The volume of this set is easily seen to be

$$
\begin{cases}\frac{1}{n !} \frac{(t-f(v))^{n}}{\gamma_{1} \gamma_{2} \cdots \gamma_{n}} & \text { if } t>f(v), \\ 0 & \text { if } t \leq f(v) .\end{cases}
$$

By the corollary, the volume of $C^{n} \cap H_{t}$ is

$$
\frac{1}{n !} \sum_{v}(-1)^{|v|} \frac{\left((t-f(v))_{+}\right)^{n}}{\gamma_{1} \cdots \gamma_{n}},
$$

where, if $y \in \mathbb{R}, y_{+}=\max \{0, y\}$, and $|v|=\sum_{i=1}^{n} \varepsilon_{i}$. This formula has already been observed in [4]. Dyer and Frieze [7] show that computing $\operatorname{vol}\left(C^{n} \cap H_{t}\right)$ is $\# P$-hard.

As another example we compute the volumes of certain projective images of the unit $n$-cube.

For $u \in \mathbb{R}^{n}$ let $T_{u}$ denote the projective transformation $T_{u}(x)=$ $x /\left(1+u^{T} x\right)$. For $u, v \in \mathbb{R}^{n}$ one has $T_{u}\left(T_{v}(x)\right)=T_{u+v}(x)$, and in particular, $T_{-u}$ is the inverse of $T_{u}$.

Let $\mathbb{R}_{+}^{n}=\left\{\left[x_{1}, \ldots, x_{n}\right]^{t} \in \mathbb{R}^{n}: x_{i} \geq 0 \quad(i=1, \ldots, n)\right\}$, the nonnegative orthant. If $u>0$, then $T_{u}$ is defined on $\mathbb{R}_{+}^{n}$. If $x \in \mathbb{R}_{+}^{n}$ and $y=T_{u}(x)$, then $0 \leq x=T_{-u}(y)=y /\left(1-u^{t} y\right)$. Clearly,

$$
T_{u}\left(\mathbb{R}_{+}^{n}\right)=\left\{y \in \mathbb{R}_{+}^{n}: u^{t} y<1\right\}
$$

This set coincides, up to the boundary, with the simplex

$$
\operatorname{conv}\left\{0, v^{(1)}, \ldots, v^{(n)}\right\}, \quad \text { where } v^{(i)}=\left[0, \ldots, 1 / u_{i}, \ldots, 0\right],
$$

the nonzero entry being in the $i$ th coordinate.

We wish to apply the corollary with the valuation $V(P)=\operatorname{vol} T_{u}(P)$, which is defined on polyhedra $P \subseteq \mathbb{R}_{+}^{n}$, to compute $V\left(C^{n}\right)$. To this end, we determine $V(F(v))$ for vertices $v=\left[\varepsilon_{1} \cdots \varepsilon_{n}\right]^{t}$ of $C^{n}$. We have

$$
\begin{aligned}
T_{u}(F(v)) & =\left\{y \in \mathbb{R}^{n}: T_{-u}(y) \in F(v)\right\} \\
& =\left\{y: y_{i} \geq 0 \text { if } \varepsilon_{i}=0, y_{i}+u^{T} y \geq 1 \text { if } \varepsilon_{i}=1, \text { and } u^{T} y \leq 1\right\} .
\end{aligned}
$$


This set coincides, up to its boundary, with the simplex

$$
\operatorname{conv}\left\{T_{u}(v), v^{(1)}, v^{(2)}, \ldots, v^{(n)}\right\},
$$

where the $v^{(i)}$,s are as before, and of course

$$
T_{u}(v)=\left[\frac{\varepsilon_{1}}{1+u^{T} v}, \ldots, \frac{\varepsilon_{n}}{1+u^{T} v}\right]^{t} .
$$

Upon observing that the determinant of the matrix

$$
\left[\begin{array}{cccc}
1 / u_{1} & & & 1 \\
& \ddots & & \vdots \\
\varepsilon_{1} /\left(1+u^{T} v\right) & \cdots & \varepsilon_{n} /\left(1+u_{n} v\right) & 1 \\
\end{array}\right]
$$

is $1 /\left(u_{1} \cdots u_{n}\left(1+u^{T} v\right)\right)$, we deduce that

$$
V(F(v))=\operatorname{vol} T_{u}(F(v))=\frac{1}{n ! u_{1} \cdots u_{n}\left(1+u^{T} v\right)} .
$$

By the corollary we have

$$
V\left(C^{n}\right)=\frac{1}{n !} \frac{1}{u_{1} \cdots u_{n}} \sum_{v} \frac{(-1)^{|v|}}{1+u^{T} v} .
$$

\section{DESCRIPTION OF THE METHOD}

Let the polytope $P$ whose volume we are to compute be given as

$$
P=\left\{x \in \mathbb{R}^{n}: x \geq 0, A x \leq b\right\},
$$

where $A$ is an $m \times n$ matrix and $b$ is a column vector in $\mathbb{R}^{m}$ having nonnegative entries. We assume that $P$ is a simple polytope and that each vertex $v$ of $P$ satisfies with equality exactly $n$ of the $m+n$ inequalities defining $P$. In particular, considering that the origin in $\mathbb{R}^{n}$ is a vertex of $P$, the entries of $b$ are positive. (This assumption can be discarded by making use of standard lexicographic techniques for handling primal degeneracy in linear programming. See [10].) Additionally, we assume the availability of a function $f(x)=c^{t} x+d$ which is constant on no edge of $P$.

For $i=1, \ldots, n$, let $r_{i}(x)$ be the residual associated with the $i$ th nonnegativity constraint; $r_{i}(x)$ is the value of the $i$ th coordinate of $x$. For $i=n+1, \ldots, m+n$, let $r_{i}(x)$ be the residual associated with the inequality involving the $(i-n)$ th row of $A$.

We can combine the above data to formulate a linear programming problem:

$$
\text { maximize } c^{t} x+d \text { subject to the constraints }
$$

$$
\begin{aligned}
A x & \leq b, \\
x & \geq 0 .
\end{aligned}
$$


The polytope $P$ is the feasible region for (2). Our assumption that each vertex of $P$ satisfies exactly $n$ of the defining inequalities with equality is the assumption of primal nondegeneracy for (2). If we consider simplex tableaux to be equivalent if they differ only by row permutation, then primal nondegeneracy implies a bijective correspondence between the set of (equivalence classes of) simplex tableaux and the vertex set of $P$.

The vertex enumerating algorithms of $[2,20,21]$ use simplex pivoting methods to obtain all of the basic feasible tableaux for $(2)$. See also $[6,22]$ for surveys of vertex-finding algorithms. Our method uses such an algorithm.

For each tableau, the numbers $e(v)$ and $\operatorname{vol}\left(F(v) \cap H_{t}\right)$ are determined, where $v$ is the vertex of $P$ corresponding to the tableau. The summation in the corollary is computed using this information.

We describe how to glean the needed information from the simplex tableaux. After introducing slack variables for (2), we have

$$
\begin{gathered}
\text { maximize }\left[\begin{array}{l}
c \\
0
\end{array}\right]^{t} \hat{x}+d \text { subject to the constraints } \\
{[A ; I] \hat{x}=b,} \\
\hat{x} \geq 0,
\end{gathered}
$$

where now $\hat{x} \in \mathbb{R}^{m+n}$. The initial tableau is

$$
T=\left[\frac{A}{A}\left|\frac{b}{-c^{t}} 0\right| \frac{b}{d}\right]
$$

corresponding to the origin in $\mathbb{R}^{n}$, a vertex of $P$. The basic sequence for $T$ is $(n+1, n+2, \ldots, n+m)$. The basic sequence for a tableau is the sequence of indices of basic columns in the order they would appear in the identity matrix.

Suppose $v$ is a vertex of $P$. Suppose $r_{k_{j}}(v)>0$ for $j=1, \ldots, m$, and $k_{1}<\cdots<k_{m}$, so that $k_{1}, \ldots, k_{m}$ are the indices of the residuals of the nonbinding constraints. Let $\widetilde{T}$ be a tableau corresponding to $v$. The entries in its basic sequence $\left(\beta_{1}, \ldots, \beta_{m}\right)$ are the numbers $k_{j}(j=1, \ldots, m)$ in some order. The tableau $\tilde{T}$ is of the form

$$
\widetilde{T}=\left[\frac{M}{\gamma_{1} \gamma_{2} \cdots \gamma_{m+n}} \mid \frac{\tilde{b}}{\tilde{d}}\right], \quad \text { where } M\left(i, \beta_{j}\right)= \begin{cases}1 & \text { if } i=j, \\ 0 & \text { if } i \neq j .\end{cases}
$$

For us, what is important is that $\gamma_{i}=0$ if and only if $i=\beta_{j}$ for some $j$, and $f(x)=\tilde{d}-\sum_{i=1}^{m+n} \gamma_{i} r_{i}(x)$. Thus, the bottom row of $\tilde{T}$ gives the coefficients of the objective function when written in terms of the residuals of the constraints which are binding at $v$. The number $e(v)$ is the number of positive $\gamma_{i}$ 's. By definition, the forward cone $F(v)$ is the set of solutions $x$ to

$$
\begin{array}{ll}
r_{i}(x)<0 & \text { if } \gamma_{i}>0, \\
r_{i}(x) \geq 0 & \text { if } \gamma_{i}<0 .
\end{array}
$$


Given a real number $t$, let $H_{t}=\left\{x \in \mathbb{R}^{n}: f(x) \leq t\right\}$, as before. We must compute the volume of the set $F(v) \cap H_{t}$. This set is given by the inequalities

$$
\begin{aligned}
& r_{i}(x)<0 \quad \text { if } \gamma_{i}>0, \\
& r_{i}(x) \geq 0 \quad \text { if } \gamma_{i}<0, \\
& \sum_{i=1}^{m+n} \gamma_{i} r_{i}(x) \geq \tilde{d}-t .
\end{aligned}
$$

This set is nonempty if $t>\tilde{d}$.

Let $i_{1}<i_{2}<\cdots<i_{n}$ be the indices of the residuals for the constraints which are binding at $v$, so that $\gamma_{i_{j}} \neq 0$ for $1 \leq j \leq n$. The volume of the set of $y=\left[y_{1}, \ldots, y_{n}\right]^{t} \in \mathbb{R}^{n}$ satisfying

$$
y_{j} \geq 0 \quad \text { for } j=1, \ldots, n, \quad \sum_{j=1}^{n}\left|\gamma_{i_{j}}\right| y_{j} \leq t-\tilde{d}
$$

is $\frac{1}{n !}(t-\tilde{d})^{n} /\left|\gamma_{i_{1}} \cdots \gamma_{i_{n}}\right|$, when $t \geq \tilde{d}$. The linear transformation mapping $x \in \mathbb{R}^{n}$ to $y=\left[-\operatorname{sgn}\left(\gamma_{i_{1}}\right) r_{i_{1}}(x), \ldots,-\operatorname{sgn}\left(\gamma_{i_{n}}\right) r_{i_{n}}(x)\right]^{t}$ maps the simplex which is the closure of the set of solutions to (4) onto the solution set to (5). We denote by $\delta_{v}$ the absolute value of the determinant of this transformation. The volume of the solution set to (4) is then

$$
\operatorname{vol}\left(F(v) \cap H_{t}\right)= \begin{cases}0 & \text { if } t \leq \tilde{d} \\ \frac{1}{n !} \frac{1}{\delta_{v}} \frac{(t-\tilde{d})^{n}}{\left|\gamma_{i_{1}} \cdots \gamma_{i_{n}}\right|} & \text { if } t>\tilde{d} .\end{cases}
$$

The number $\delta_{v}$ in (6) is easily seen to be the determinant of the basis matrix - the matrix consisting of the columns of $[A: I]$ having indices basic in $\widetilde{T}$ and occurring in the order dictated by the basic sequence for $\widetilde{T}$. It is easy to calculate $\delta_{v}$ if we have arrived at $\widetilde{T}$ from $T$ by a sequence of pivots. It is the product of the pivot elements.

Finally, upon multiplying both sides of $(6)$ by $(-1)^{e(v)}$, we get

$$
(-1)^{e(v)} \operatorname{vol}\left(F(v) \cap H_{t}\right)= \begin{cases}0 & \text { if } t \leq \tilde{d}, \\ \frac{(-1)^{n}}{n !} \frac{1}{\delta_{v}} \frac{(t-\tilde{d})^{n}}{\gamma_{i_{1}} \cdots \gamma_{i_{n}}} & \text { if } t>\tilde{d} .\end{cases}
$$

Summing these numbers for each vertex $v$ yields the volume of the set $P \cap H_{t}$. If $t$ exceeds the optimal value of the linear programming problem (2), then the sum is the volume of $P$.

Observe that for large $t$ the functions of $t$ that we sum are polynomials, and the sum is a constant-the volume of $P$. It follows that the sum is a constant polynomial. Evaluation at $t=0$ yields the volume of $P$ as the sum of the numbers

$$
N_{v}=\frac{1}{n !} \frac{1}{\delta} \frac{\tilde{d}^{n}}{\delta_{i_{1}} \cdots \gamma_{i_{n}}}
$$




\section{AN EXAMPLE AND COMMENTS}

Figures 1 and 2 exhibit the feasible tableaux for the problem

$$
\begin{aligned}
\operatorname{maximize} & x_{1}+x_{2} \\
\text { subject to }-x_{1}+x_{2} & \leq 2, \\
x_{2} & \leq 4, \\
3 x_{1}+2 x_{2} & \leq 15, \\
x_{1}, x_{2} & \geq 0,
\end{aligned}
$$

along with a graph indicating the corresponding vertices. Our computations, as shown, indicate that the area of the polygon is $38 / 3$ (which, in this example, can easily be checked by other means).

$$
\begin{aligned}
& I \quad\left[\begin{array}{ccccc|c}
-1 & 1 & 1 & 0 & 0 & 2 \\
0 & 1 & 0 & 1 & 0 & 4 \\
3 & 2 & 0 & 0 & 1 & 15 \\
\hline-1 & -1 & 0 & 0 & 0 & 0
\end{array}\right] \\
& \text { II }\left[\begin{array}{ccccc|c}
0 & 5 / 3 & 1 & 0 & 1 / 3 & 7 \\
0 & 1 & 0 & 1 & 0 & 4 \\
1 & 2 / 3 & 0 & 0 & 1 / 3 & 5 \\
\hline 0 & -1 / 3 & 0 & 0 & 1 / 3 & 5
\end{array}\right] \\
& \text { I I }\left[\begin{array}{ccccc|c}
0 & 0 & 1 & -5 / 3 & 1 / 3 & 1 / 3 \\
0 & 1 & 0 & 1 & 0 & 4 \\
1 & 0 & 0 & -2 / 3 & 1 / 3 & 7 / 3 \\
\hline 0 & 0 & 0 & 1 / 3 & 1 / 3 & 19 / 3
\end{array}\right. \\
& \text { IV }\left[\begin{array}{ccccc|c}
0 & 0 & 3 & -5 & 1 & 1 \\
0 & 1 & 0 & 1 & 0 & 4 \\
1 & 0 & -1 & 1 & 0 & 2 \\
\hline 0 & 0 & -1 & 2 & 0 & 6
\end{array}\right. \\
& \text { V }\left[\begin{array}{ccccc|c}
5 & 0 & -2 & 0 & 1 & 11 \\
-1 & 1 & 1 & 0 & 0 & 2 \\
1 & 0 & -1 & 1 & 0 & 2 \\
\hline-2 & 0 & 1 & 0 & 0 & 2
\end{array}\right] \\
& \delta_{v}=1 . \quad N_{v}=\frac{1}{2 !} \quad \frac{1}{1} \quad \frac{0^{2}}{(-1)(-1)} . \\
& \delta_{v}=3 . \quad N_{v}=\frac{1}{2 !} \quad \frac{1}{3} \quad \frac{5^{2}}{(-1 / 3)(1 / 3)} \text {. } \\
& \delta_{\mathrm{v}}=3 . \quad \mathrm{N}_{\mathrm{v}}=\frac{1}{2 !} \quad \frac{1}{3} \quad \frac{(19 / 3)^{2}}{(1 / 3)(1 / 3)} . \\
& \delta_{v}=1 . \quad N_{v}=\frac{1}{2 !} \quad \frac{1}{1} \quad \frac{6^{2}}{(-1)(2)} \\
& \delta_{\mathrm{v}}=1 . \quad \mathrm{N}_{\mathrm{v}}=\frac{1}{2 !} \quad \frac{1}{1} \quad \frac{2^{2}}{(-2)(1)} . \\
& \sum N_{v} \quad=\quad \frac{38}{3}
\end{aligned}
$$




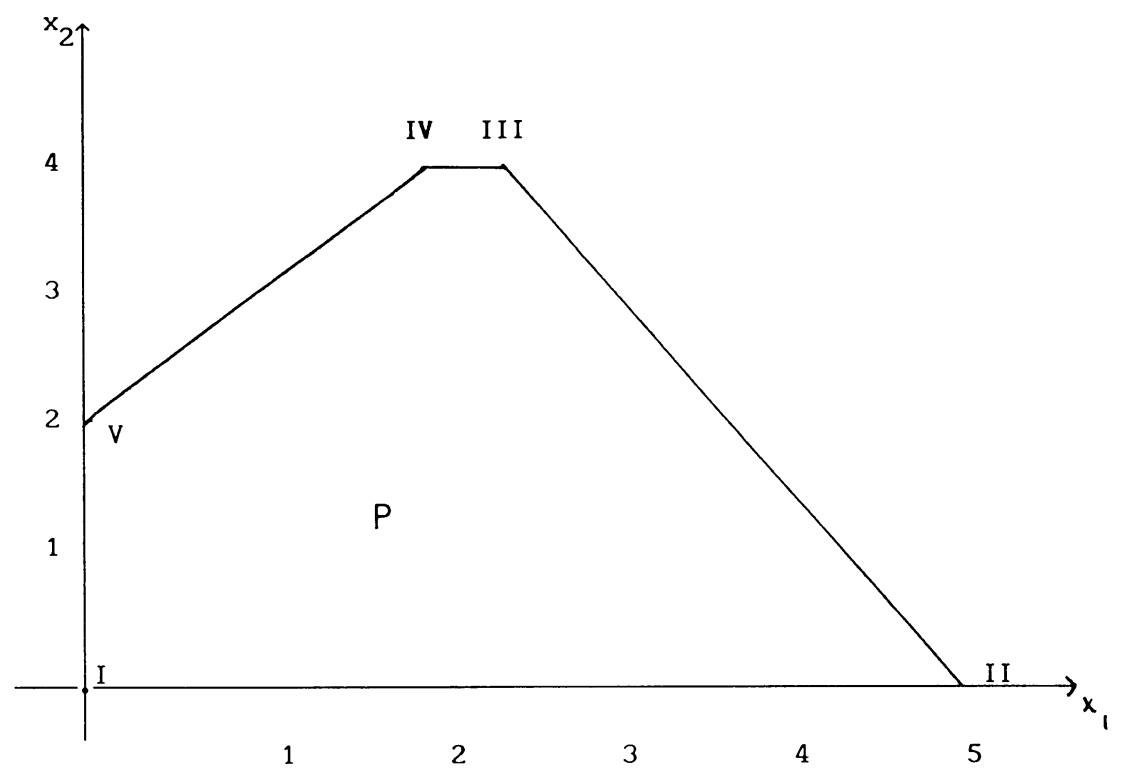

FIGURE 2

The main contributor to the complexity of this method is the possibly high number of vertices of the polytope $P$. A polytope of dimension $n$ determined by $m+n$ linear inequality constraints may have as many as $\left(\begin{array}{c}m+n-\lfloor(n+1) / 2\rfloor \\ 2\end{array}\right)+$ $\left(\begin{array}{c}m+n-\lfloor(n+2) / 2\rfloor \\ m\end{array}\right)$ vertices (see [19]).

A problem which provides a complication in higher dimensions is that of round-off error. The method requires summing a lot of numbers, some positive and some negative. These numbers, compared to the volume of $P$, can be quite large in magnitude, so that there can be considerable loss of significance. One (perhaps costly) way around this is the use of "exact arithmetic." To illustrate the extent to which this approach can indeed be costly and to provide a negative solution to the problem of Dyer and Frieze [7] mentioned in the Introduction, we consider again the example at the end of $\S 2$, with

$$
u=\left[\frac{1}{2}, \frac{1}{4}, \ldots, \frac{1}{2^{n}}\right]^{t}
$$

The projective image $T_{u}\left(C^{n}\right)$ is the polytope which consists of those $y \in \mathbb{R}^{n}$ which satisfy the $2 n$ inequalities

$$
\begin{gathered}
y_{i} \geq 0 \quad(\text { for } 1 \leq i \leq n), \\
\left(1+\frac{1}{2}\right) y_{1}+\frac{1}{4} y_{2}+\cdots+\frac{1}{2^{n}} y_{n} \leq 1,
\end{gathered}
$$




$$
\begin{aligned}
& \frac{1}{2} y_{1}+\left(1+\frac{1}{4}\right) y_{2}+\cdots+\frac{1}{2^{n}} y_{n} \leq 1, \\
& \frac{1}{2} y_{1}+\frac{1}{4} y_{2}+\cdots+\left(1+\frac{1}{2^{n}}\right) y_{n} \leq 1 .
\end{aligned}
$$

Its volume is

$$
\frac{1}{n ! u_{1} \cdots u_{n}} \sum_{\substack{v, \mathrm{a} \\ \text { vertex of } C^{n}}} \frac{(-1)^{|v|}}{1+u^{t} v}=\frac{2^{\left(n^{2}+3 n\right) / 2}}{n !} \sum_{N=2^{n}}^{2^{n+1}-1} \frac{(-1)^{\eta(N)-1}}{N}
$$

where $\eta(N)$ is the number of 1's in the binary expansion of $N$. Suppose this number, written as a reduced fraction, is $a / b$. Note that each prime $N$ such that $2^{n}<N<2^{n+1}$ divides $b$, so a very crude lower bound on $b$ is $2^{k}$, where $k$ is the number of such primes $N$. It follows by the prime number theorem $[12$, p. 9$]$ that $k$ is not bounded by a polynomial in $n$. We see that the number of digits in the binary expansion of $b$ is not bounded by a polynomial in $n$.

In the presence of primal degeneracy there is no longer a bijective correspondence between the set of vertices of $P$ and the set of equivalence classes of feasible tableaux. In this case it is nevertheless possible to find the desired volume by performing the summation, but now over the set of tableaux for which a lexicographic positivity condition holds.

The requirement that the objective function $f$ be nonconstant on the edges of $P$ also provides a complication. This requirement is fulfilled by $f(x)=c^{t} x$, where $c=\left[1, M, \ldots, M^{n-1}\right]^{t}$, for $M$ a sufficiently large number. If $A$ and $b$ have rational entries, then one can show (using the methods of [24, §11.3]) that $M$ can be chosen to be of size polynomial in the size of $(A, b)$.

\section{ACKNOWLEDGMENT}

We wish to note our appreciation for help and encouragement rendered by several people-Javier Bernal, Mark Hartmann, Victor Klee, Carl Lee, Walter Morris, Ted Speevak, and Christoph Witzgall. Aid rendered ranged from encouraging the writing of the paper through providing references of which I was unaware, to reading a preliminary draft, finding mistakes, and suggesting improvements!

\section{BIBLIOGRAPHY}

1. E. L. Allgower and P. M. Schmidt, Computing volumes of polyhedra, Math. Comp. 46 (1986), 171-174.

2. M. L. Balinski, An algorithm for finding all vertices of convex polyhedral sets, SIAM J. Appl. Math. 9 (1961), 72-88.

3. J. Bárány and Z. Füredi, Computing the volume is difficult, Proc. 18th Annual ACM Sympos. on Theory of Computing, 1986, pp. 442-447.

4. D. L. Barrow and P. W. Smith, Spline notation applied to a volume problem, Amer. Math. Monthly 86 (1979), 50-51.

5. J. Cohen and T. Hickey, Two algorithms for determining volumes of convex polyhedra, J. Assoc. Comput. Mach. 26 (1979), 401-414.

6. M. E. Dyer, The complexity of vertex enumeration methods, Math. Oper. Res. 8 (1983), $381-402$. 
7. M. E. Dyer and A. M. Frieze, On the complexity of computing the volume of a polyhedron, SIAM J. Comput. 17 (1988), 967-974.

8. H. G. Eggleston, Convexity, Cambridge Univ. Press, 1958.

9. G. Elekes, A geometric inequality and the complexity of computing volume, Discrete Comput. Geom. 1 (1986), 289-292.

10. S. I. Gass, Linear programming, McGraw-Hill, 1958.

11. B. Grünbaum, Convex polytopes, Interscience, 1967.

12. G. H. Hardy and E. M. Wright, An introduction to the theory of numbers, fourth ed., Clarendon Press, 1968.

13. J. B. Lasserre, An analytical expression and an algorithm for the volume of a convex polyhedron in $\mathbb{R}^{n}$, J. Optim. Theory Appl. 39 (1983), 363-377.

14. J. Lawrence, Valuations and polarity, Discrete Comput. Geom. 3 (1988), 307-324.

15. Y. T. Lee and A. A. G. Requicha, Algorithms for computing the volume and other integral properties of solids. I. Known methods and open issues, Comm. ACM 25 (1982), 635-641.

16. _ Algorithms for computing the volume and other integral properties of solids. II. A family of algorithms based on representation conversion and cellular approximation, Comm. ACM 25 (1982), 642-650.

17. L. Lovász, An algorithmic theory of numbers, graphs and convexity, SIAM, 1986.

18. P. McMullen, Angle-sum relations for polyhedral sets, Mathematika 33 (1986), 173-188.

19. P. McMullen and G. C. Shephard, Convex polytopes and the upper bound conjecture, Cambridge Univ. Press, 1971.

20. M. Manas and J. Nedoma, Finding all vertices of a convex polyhedron, Numer. Math. 12 (1968), 226-229.

21. T. H. Mattheiss, An algorithm for determining irrelevant constraints and all vertices in systems of linear inequalities, Oper. Res. 21 (1973), 247-260.

22. T. H. Mattheis and D. S. Rubin, $A$ survey and comparison of methods for finding all vertices of convex polyhedral sets, Math. Oper. Res. 5 (1980), 167-185.

23. G. C. Rota, The valuation ring, Studies in Pure Mathematics (L. Mirsky, ed.), Academic Press, New York, 1971.

24. A. Schrijver, Theory of linear and integer programming, Wiley, Chichester, 1986.

25. G. C. Shephard, An elementary proof of Gram's theorem for convex polytopes, Canad. J. Math. 19 (1967), 1214-1217.

26. D. P. Shoemaker and T. C. Huang, A systematic method for calculating volumes of polyhedra corresponding to Brillouin zones, Acta Cryst. Sect. A 7 (1954), 249-259.

27. T. Speevak, An efficient algorithm for obtaining the volume of a special kind of pyramid and application to convex polyhedra, Math. Comp. 46 (1986), 531-536.

28. J. Stoer and C. Witzgall, Convexity and optimization in finite dimensions, Springer-Verlag, 1970.

29. L. G. Valiant, The complexity of enumeration and reliability problems, SIAM J. Comput. 8 (1979), 410-421.

30. B. Von Hohenbalken, Finding simplicial subdivisions of polytopes, Math. Programming 21 (1981), 233-234.

Department of Mathematical Sciences, George Mason University, Fairfax, Virginia 22030

Center for Computing and Applied Mathematics, National Institute of Standards and Technology, Gaithersburg, Maryland 20899

E-mail address: lawrence@gmuvax.gmu.edu 